\title{
The Propagation Characteristics of Wave-Guiding Structures with Very Thin Superconductors; Application to Coplanar Waveguide $\mathrm{YBA}_{2} \mathrm{Cu}_{3} \mathrm{O}_{7-x}$ Resonators
}

\author{
Boele B. G. Klopman, Gerrit J. Gerritsma, and Horst Rogalla
}

\begin{abstract}
We have analyzed the propagation characteristics of wave-guiding structures with superconductors which are thin compared to the magnetic penetration depth. The complex propagation constant is evaluated within the framework of the modified spectral domain method without the need for numerical calculations in the complex plane. Good agreement is found with the results of other methods. The numerical analysis is instrumental in deducing results for the penetration depth and the surface resistance of $\mathrm{YBa}_{2} \mathrm{Cu}_{3} \mathrm{O}_{7-x}$ thin films on sapphire with a $\mathrm{PrBa}_{2} \mathrm{Cu}_{3} \mathrm{O}_{7-x}$ buffer layer. We confirm recent observations of a non-single-gap BCS temperature dependence.
\end{abstract}

\section{INTRODUCTION}

$\mathbf{T}$ HE SURFACE impedance of superconductors is characterized by a low resistance and a relatively high reactance. This makes superconductors suitable for microwave applications, which are impossible if normal conductors are used. As a result of the low surface resistance, the power losses in superconductors are low, which offers the possibility of low-loss filters with a sharp frequency response [1], [2]. The surface reactance represents the stored energy in the superconductor. If the stored energy is increased at a constant level of transmitted microwave power, the phase velocity is reduced, since the energy flux remains the same. Therefore, the surface reactance has the effect of slowing down the electromagnetic wave. Transmission lines exhibit a high slowing factor if the geometry favors the stored energy relative to the transmitted energy. The stored kinetic energy of the charge carriers in a superconducting film increases as the thickness of the film is reduced below the magnetic penetration depth. This is a consequence of the increased current density necessary to support the same current or magnetic field. The transmitted energy on the other hand is reduced by choosing a small geometry. In this way very compact microwave devices, such as filters and delay lines, can be fabricated [3], [4]. The miniaturization is also made possible by the low surface resistance. In addition, the influence of the surface reactance also can be used to determine the penetration depth.

Manuscript received March 16, 1992; revised August 31, 1992.

The authors are with the University of Twente, Department of Applied Physics, P.O. Box 217, 7500 AE Enschede, The Netherlands.

IEEE Log Number 9207421.
Both the design of microwave devices and the characterization of the superconductors rely on the accurate calculation of the propagation characteristics of superconducting transmission lines. Superconducting microstrip lines with very slow phase velocities have been analyzed by a spectral domain method, modified by complex resistive boundary conditions [5]. The modification is necessary because of the coupling of the electromagnetic field from one side of the film to the other side. We will show that the complex propagation constant (attenuation and wave number) of different waveguiding structures can be evaluated within the framework of the modified spectral domain method without numerical calculations in the complex plane. Expressed in transmission line components, this corresponds to the calculation of the resistance and kinetic inductance of the structure. The numerical analysis is applied to coplanar waveguide transmission lines.

The suitability of the method is demonstrated by the investigation of experimental $\mathrm{YBa}_{2} \mathrm{Cu}_{3} \mathrm{O}_{7-x}$ coplanar waveguide resonators. It is not possible to obtain a high slowing factor using a coplanar waveguide with reasonable dimensions. The uniplanar metallization makes the transmitted energy much less sensitive to the separation of the strips than in the case of microstrip lines. However the effect of the surface reactance on the resonance frequency of the resonator is significant enough to determine the penetration depth. Results for the surface resistance are obtained from the observed quality factor of the resonator. Investigations of the surface impedance (surface resistance and penetration depth) are of importance, since these can help to clarify the nature of the high-temperature superconductors. The lack of a detailed picture of the couplingmechanism of the electrons in these superconductors is accompanied by observations of non-BCS behavior [6], [7].

We start with an analysis of the surface impedance of a superconducting or normal film of arbitrary thickness. This also naturally leads to the complex resistive boundary conditions in the case of very thin films.

\section{THEORY}

\section{A. Surface Impedance of a Film of Arbitrary Thickness}

To investigate the surface impedance of a superconducting film, we first demonstrate how the Maxwell equations have 
to be modified, assuming the two-fluid model and the London equations give a valid description of the superconductor. Despite the phenomenological nature, much of the characteristic features of the electrodynamics is easily visualized by this approach, at least qualitively.

The electrons in a superconductor can be divided in normal and superconducting electrons, according to the two-fluid model. Therefore the total current density $\boldsymbol{J}=\boldsymbol{J}_{n}+\boldsymbol{J}_{s}$ consists of a dissipative normal part $\boldsymbol{J}_{n}$ and a superconducting dissipationless part $\boldsymbol{J}_{s}$. The normal part $\boldsymbol{J}_{n}$ corresponds to the conductivity of the normal electrons:

$$
\boldsymbol{J}_{n}=\sigma_{n} \boldsymbol{E},
$$

giving rise to power losses. The superconducting electrons are described by the London equation [8]:

$$
\frac{\partial \boldsymbol{J}_{s}}{\partial t}=\frac{\boldsymbol{E}}{\mu_{0} \lambda^{2}},
$$

which represents the free acceleration of the electrons in an electric field. The magnetic penetration depth is denoted by $\lambda$.

For time-harmonic electric fields, (1) and (2) can be incorporated in the first Maxwell equation $\nabla \times \boldsymbol{H}=y \boldsymbol{E}$, provided $y$ is written as:

$$
y=\sigma+j \omega \varepsilon .
$$

Here the complex conductivity is defined as:

$$
\sigma=\sigma_{n}-j \sigma_{s},
$$

with:

$$
\sigma_{s}=\frac{1}{\omega \mu_{0} \lambda^{2}} .
$$

In (3) the last term represents the displacement current. The case of a normal conductor is obtained by setting $\sigma_{s}=0$.

The second Maxwell equation $\nabla \times \boldsymbol{E}=-z \boldsymbol{H}$ remains unchanged:

$$
z=j \omega \mu_{0} .
$$

So the two-fluid model and the London equations lead to the introduction of a complex conductivity. Although this complex conductivity is a result of the phenomenological London equations, a comparison can be made with more theoretically based results for the complex conductivity [9]. This yields values for $\sigma_{n}$ and $\lambda$ on the basis of the BCS theory. It is also possible to use the phenomenological Gorter-Casimir expressions [10] for $\sigma_{n}$ and $\lambda$.

The interpretation of the complex conductivity is made clear by manipulating the two Maxwell equations to obtain for the supplied time-averaged complex power density $\bar{p}_{s}$ :

$$
\begin{aligned}
\bar{p}_{s} & =-\frac{1}{2} \nabla \cdot\left(\boldsymbol{E} \times \boldsymbol{H}^{*}\right) \\
& =\frac{1}{2} \sigma_{n}|\boldsymbol{E}|^{2}+\frac{1}{2} j \omega \mu_{0}\left(\lambda^{2}\left|\boldsymbol{J}_{s}\right|^{2}+|\boldsymbol{H}|^{2}\right)-\frac{1}{2} j \omega \varepsilon|\boldsymbol{E}|^{2} \\
& =\bar{p}_{d}+2 j \omega\left(\bar{w}_{K}+\bar{w}_{M}-\bar{w}_{E}\right) .
\end{aligned}
$$

So the normal (real) part of the conductivity corresponds to a dissipated power density $\bar{p}_{d}$. while the superconducting (imaginary) part is related to the kinetic energy density $\bar{w}_{K}$ of the superconducting electrons. In (7) $\bar{w}_{M}$ and $\bar{w}_{E}$ are the stored magnetic and electric energy densities (all quantities time-averaged)

Expression (7) will be used to evaluate the kinetic and magnetic inductance of an infinite film of arbitrary thickness $t$. First we develop expressions related to the complex power. In the following the displacement current will be neglected, which is a good approximation for most conductors at microwave frequencies.

We consider plane waves, propagating in the $z$-direction, perpendicular to the film. The film boundaries are located at $z=0$ and $z=t$. The magnetic and electric field in the interior of the film are given by [11]:

$$
\begin{gathered}
H_{y}(z)=H_{01} e^{-j k z}+H_{02} e^{j k z}, \\
E_{x}(z)=\eta\left(H_{01} e^{-j k z}-H_{02} e^{j k z}\right) . \\
0 \leq z \leq t
\end{gathered}
$$

The wave number $k$ and the intrinsic impedance $\eta$ follow from the Maxwell equations:

$$
\begin{aligned}
& k=\sqrt{-z y}, \\
& \eta=\sqrt{z / y} .
\end{aligned}
$$

In the case of a normal conductor this yields:

$$
\begin{aligned}
k & =\sqrt{\omega \mu_{0} \sigma_{n} / 2}(1-j) \\
& =(1-j) / \delta, \\
\eta & =\sqrt{\omega \mu_{0} / 2 \sigma_{n}}(1+j) \\
& =\frac{1}{2} \omega \mu_{0} \delta(1+j),
\end{aligned}
$$

where $\delta$ is the classical skin depth:

$$
\delta=\sqrt{2 / \omega \mu_{0} \sigma_{n}} .
$$

For a superconductor $k$ and $\eta$ are given by (assuming $\left.\sigma_{n} \ll \sigma_{s}\right)$ :

$$
\begin{aligned}
k & =\sqrt{\omega \mu_{0} \sigma_{s}}\left(\sigma_{n} / 2 \sigma_{s}-j\right) \\
& =\left((\lambda / \delta)^{2}-j\right) / \lambda, \\
\eta & =\sqrt{\omega \mu_{0} / \sigma_{s}}\left(\sigma_{n} / 2 \sigma_{s}+j\right) \\
& =\omega \mu_{0} \lambda\left((\lambda / \delta)^{2}+j\right) .
\end{aligned}
$$

We will see that $R_{s}=\operatorname{Re}(\eta)$ is related to power losses, while $X_{s}=\operatorname{Im}(\eta)$ is a measure of the stored energy. So in superconductors the power losses are usually much smaller than the stored energy $\left(\lambda \ll \delta\right.$ for $\left.\sigma_{n} \ll \sigma_{s}\right)$. In addition, in this case the power losses are much smaller than in a normal conductor.

Invoking tangential continuity of $\boldsymbol{H}$ at the boundaries, $H_{y}(0)=H_{1}, H_{y}(t)=H_{2}$, yields:

$H_{y}(z)=\left[H_{1} \sin (k(t-z))+H_{2} \sin (k z)\right] / \sin (k t)$,

$$
E_{x}(z)=\eta\left[H_{1} \cos (k(t-z))-H_{2} \cos (k z)\right] / j \sin (k t) .
$$


The complex power supplied to the film per unit area is evaluated as:

$$
\begin{aligned}
P & =\frac{1}{2} E_{x}(0) \times H_{y}^{*}(0)-\frac{1}{2} E_{x}(t) \times H_{y}^{*}(t) \\
& =\frac{1}{2} Z_{s 1}\left|H_{1}-H_{2}\right|^{2}+\frac{1}{2} Z_{s 2}\left(\left|H_{1}\right|^{2}+\left|H_{2}\right|^{2}\right),
\end{aligned}
$$

where the effective surface impedances $Z_{s 1}$ and $Z_{s 2}$ are defined:

$$
\begin{aligned}
& Z_{s 1}=\frac{\eta}{j \sin (k t)}, \\
& Z_{s 2}=\eta \frac{\cos (k t)-1}{j \sin (k t)} .
\end{aligned}
$$

Two limiting cases are of particular interest. Firstly, for a very thin film $(|k t| \ll 1$, i.e., $t \ll \lambda, \delta)$ we have:

$$
\begin{array}{ll}
Z_{s 1}=\frac{\eta}{j k t}=\frac{1}{\sigma t}, & \\
Z_{s 2}=\frac{1}{2} j \omega \mu_{0} t, & (t \ll \lambda, \delta)
\end{array}
$$

while in the case of a relatively thick film:

$$
Z_{s 1}=0, Z_{s 2}=\eta \quad(t \gg \lambda, \delta)
$$

Thus the second term in (19) is dominant if $t \gg \lambda, \delta$, which actually is the bulk material case. Indeed we find the well-known expression for the complex power [11], with the addition that the magnetic field at both film boundaries contributes. In this case the surface impedance equals the intrinsic wave impedance. For $t \ll \lambda, \delta$ only the first term in (19) survives. In this case the impedance depends inversely proportional on the thickness of the film. To be specific:

$$
\begin{aligned}
& R_{s 1}=R_{s} 2 \lambda / t, \quad(t \ll \lambda, \delta) \\
& X_{s 1}=X_{s} \lambda / t .
\end{aligned}
$$

Here $R_{s}$ and $X_{s}$ denote the surface resistance and reactance for bulk material: $\eta=R_{s}+j X_{s}$. So for very thin films, the effective surface resistance is by a factor $2 \lambda / t$ higher than the bulk value, the surface reactance by a factor $\lambda / t$. This correction should be kept in mind when data for very thin films are to be interpreted.

In the intermediate range of thicknesses, the complex power depends on the exact values of $H_{1}$ and $H_{2}$. In general no overall impedance can be defined uniquely. An exception occurs if the field is confined to one side of the film $\left(H_{1}\right.$ or $H_{2}=0$ ), meaning that the film represents a real wall. Then the surface impedance reads as:

$$
Z_{s}=-j \eta \cot (k t),\left(H_{1} \text { or } H_{2}=0\right)
$$

which is in agreement with the usual expression for this case [12].

The general pattern of $Z_{s 1}$ and $Z_{s 2}$ is shown in Fig. 1 at a frequency $f=10 \mathrm{GHz}$, with a penetration depth $\lambda=100 \mathrm{~nm}$ and skin depth $\delta=100 \lambda$. Clearly, $Z_{s 1}$ reaches its limiting value (see (24), (25)) not far below $t / \lambda=1$. For $Z_{s 2}$ to

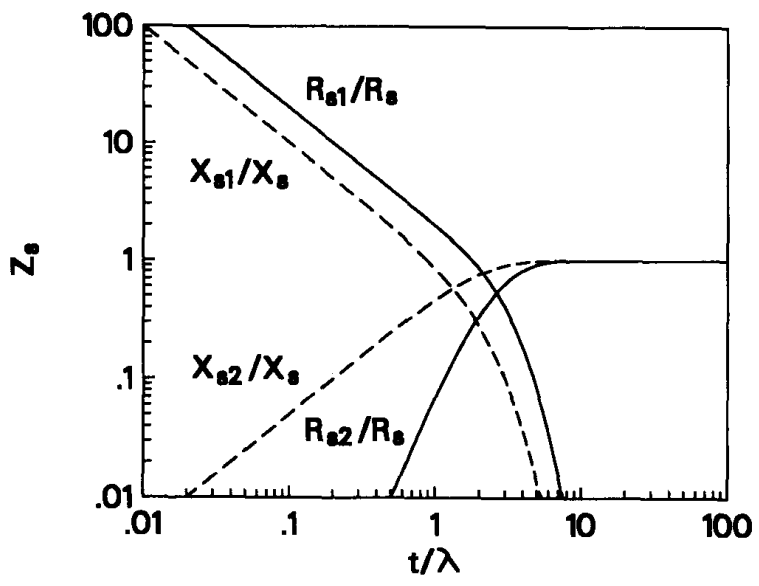

Fig. 1. The effective surface impedances $Z_{s 1}=R_{s 1}+j X_{s 1}$ (dominan if $t \ll \lambda$ ) and $Z_{s 2}=R_{s 2}+j X_{s 2}$ (dominant if $t \gg \lambda$ ) as a function of $t / \lambda$. The resistive and reactive parts are normalized to the quantities of bulk material $(t \gg \lambda)$

reach its limiting value (23) for $t>\lambda$ a somewhat stronger condition on $t / \lambda$ is required; depending on the acceptable errors, the value of $t / \lambda$ can be as high as approximately 5 . The difference in the asymptotic behavior of $Z_{s 1}$ and $Z_{s 2}$ can be understood, recalling that the current density at both sides of the film falls off as $\exp (-d / \lambda)$ inside the conductor, where $d$ is the distance from the boundary of the film. As soon as $t$ becomes comparable to $\lambda$ or smaller, the current density inside the film is almost uniform. However, if the current density is to die out in the interior of the conductor, the distance to both sides of the film should be several penetration depths.

We now turn to the calculation of the kinetic inductance $L_{K}$ and the magnetic inductance $L_{M}$ for an infinite film. Per unit length those inductances are related to the time-averaged kinetic and magnetic energy density through (cf. equation (7))

$\frac{1}{4}\left(L_{K}+L_{M}\right)|I|^{2}=\bar{W}_{K}+\bar{W}_{M}=\frac{1}{2 \omega} \iint \operatorname{Im}\left(\bar{p}_{s}\right) d y d z$.

Here $I$ is the amplitude of the total current through the film:

$$
I=\iint J_{s} d y d z=\left(H_{1}-H_{2}\right) w,
$$

where $w$ is the width of the film. From (27) we obtain for $L_{K}$ and $L_{M}$ :

$$
\begin{aligned}
& L_{K}=\mu_{0} \lambda^{2} \iint\left|J_{s}\right|^{2} d y d z / I^{2}, \\
& L_{M}=\mu_{0} \iint|H|^{2} d y d z / I^{2} .
\end{aligned}
$$

Again two limiting cases are considered. The inductances $L_{K}$ and $L_{M}$ are equal for $t \gg \lambda, \delta$, because (neglecting $\sigma_{n}$ ):

$$
\left|\frac{H}{J_{s}}\right|^{2}=\left|\frac{H}{E}\right|^{2}\left|\frac{E}{J_{s}}\right|^{2}=|\eta \sigma|^{-2}=\lambda^{2} .
$$

Evaluating the integrals yields in this transversely uniform case:

$$
L_{K}=L_{M}=\frac{\mu_{0} \lambda}{2 w} . \quad(t \gg t, \delta)
$$


The property of equal $L_{K}$ and $L_{M}$ is generally valid for bulk superconductors [13]. For a very thin film on the other hand, we obtain for $L_{K^{r}}$ :

$$
L_{K}=\frac{\mu_{0} \lambda^{2}}{w t}, \quad(t \ll \lambda, \delta)
$$

while $L_{M}$ approaches zero. Thus in this range of thicknesses $L_{K}$ is a factor $2 \lambda / t$ higher than in the case of relatively thick films. The increased value of $L_{K}$ results in a higher slowing factor of the electromagnetic waves. The accompanying increase in the losses is usually not a problem, since $R_{s}$ is very low for superconductors, cf. (24). Structures with a high slowing factor contribute to the realization of very compact microwave circuits [3], [4]. In addition, by measuring the slowwave behavior it is possible to determine $\lambda$, because of the role it plays in $L_{K}$.

\section{B. Propagation Characteristics of Wave-Guiding Structures with Non-Perfect Conductors}

In commonly used wave-guiding structures, we are not dealing with plane waves, as was supposed in the previous section. In general the modes are at best quasi-TEM, but generally hybrid of nature. We will analyze the influence of a non-perfect conducting (i.e., normal or superconducting) metallization with the restriction to the case of very thin films, where the thickness $t$ of the metallization is much smaller than the penetration depth $\lambda$ and the skin depth $\delta: t \ll \lambda, \delta$. In this limit it can be shown on the basis of (17) and (18) that the boundary conditions for plane waves can be expressed as:

$$
\begin{aligned}
& \boldsymbol{E}_{1}=\boldsymbol{E}_{2}=\boldsymbol{R} \boldsymbol{j}, \\
& \boldsymbol{n} \times\left(\boldsymbol{H}_{1}-\boldsymbol{H}_{2}\right)=\boldsymbol{j},
\end{aligned}
$$

with:

$$
R=\frac{1}{\sigma t}=Z_{s 1}
$$

The subscripts 1 and 2 refer to both sides of the film. The quantity $j$ denotes the total current density per unit length through the cross-section of the film, $\boldsymbol{n}$ is a unit normal vector to the film.

It is assumed that the complex resistive boundary conditions (34) and (35) locally remain valid for non-plane waves. This is an approximation, especially at the sharp comers of the metallization. However, the method has been proved to be quite accurate [5].

The spectral domain method can be applied for a full wave analysis of different wave-guiding structures, such as coplanar waveguide transmission lines (Fig. 2) [14]. The fact that the metallization strips are non-perfect very thin conductors is taken into account by a modification of this method through the complex resistive boundary conditions (34) and (35), as pointed out by Pond et al. [5]. Subsequent application of the modified spectral domain method can be found in several works [15], [16].

In short, the electric field at the strips interface $\left(x=h_{1}+h_{2}\right)$ is expressed in terms of the current density in the strips by means of the so-called dyadic Green's function. The Green's function corresponds to the case of perfect conductors of zero

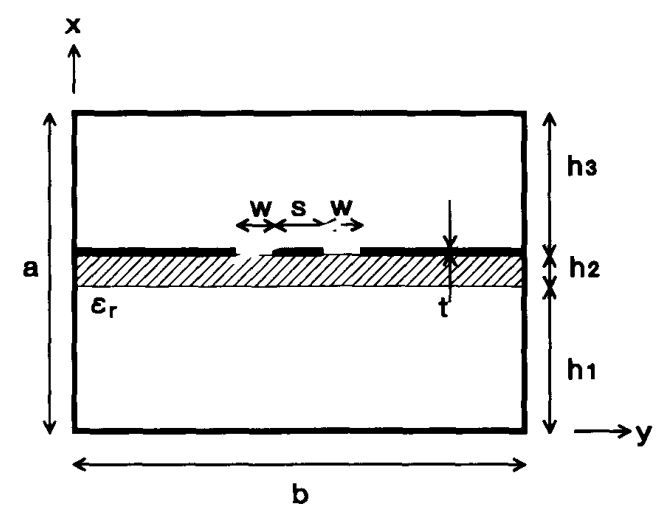

Fig. 2. Geometry of a coplanar waveguide enclosed in a box with strips of thickness $t$. Unless otherwise stated, the spectral domain calculations are performed at a frequency $f=10 \mathrm{GHz}$ with geometrical parameters $\varepsilon_{r}=10$, and $a=18, b=10, s=0.5, w=0.2, h_{1}=9, h_{2}=0.635$, and $h_{3}=8.365 \mathrm{~mm}$.

thickness. In this limit the edge condition [17] implies that the tangential field and current components behave like $\rho^{-1 / 2}$ for vanishing radial distance $\rho$ from the edge. Invoking boundary condition (34) establishes a relation between the electric field in the slots and the currents in the strips. The electric field in the slots is expanded into a set of basis functions. If these basis functions are chosen to satisfy the edge condition, normally only a few basis functions are needed for an accurate description of the field pattern. The drawback of this approach is the appearance of a logarithmic divergence for quantities which involve the integration of the squared field or current components [18], [19]. In the presentation of the numerical results we will discuss this problem in more detail.

The electric field in the slots and the currents in the strips are non-zero in complementary regions. This means that Galerkin's method can be applied to obtain a homogeneous system of linear equations with the weighting factors in the series of basis functions as unknown coefficients [20]. The propagation constant $\gamma$ of the structure corresponds with non-trivial solutions of this system of equations, meaning a determinant of zero:

$$
\operatorname{det}\left[\bar{Z}\left(j \gamma, j Z_{s 1}\right)\right]=0
$$

Here, $\bar{Z}$ denotes the matrix corresponding to the system of equations.

In general the propagation constant is a complex number with attenuation constant $\alpha$ and wave number $\beta: \gamma=\alpha+j \beta$. This would imply the use of a root finding algorithm in the complex plane. However, in the lossless case $\left(R_{s 1}=0\right.$ and $\alpha=0$ ) the determinant is real, as is emphasized by the arguments $j \gamma$ and $j Z_{s 1}$. Then (37) becomes:

$$
\operatorname{det}\left[\bar{Z}\left(-\beta,-X_{s 1}\right)\right]=0 .
$$

In the case of a small amount of conductor losses in the strips, it is also possible to calculate the attenuation constant $\alpha$ without explicit complex numerical computation. This is demonstrated as follows. If the losses are low, the solution of 
(37) can be approximated by:

$$
\begin{aligned}
0= & \operatorname{det}\left[\overline{\boldsymbol{Z}}\left(-\beta+j \alpha,-X_{s 1}+j R_{s 1}\right)\right] \\
\simeq & \operatorname{det}\left[\overline{\boldsymbol{Z}}\left(-\beta,-X_{s 1}\right)\right]+\frac{\partial \operatorname{det}\left[\overline{\boldsymbol{Z}}\left(-\beta,-X_{s 1}\right)\right]}{\partial(-\beta)} j \alpha \\
& +\frac{\partial \operatorname{det}\left[\overline{\boldsymbol{Z}}\left(-\beta_{+}-X_{x 1}\right)\right]}{\partial\left(-X_{s 1}\right)} j R_{s 1} .
\end{aligned}
$$

Here the first term in the right hand side corresponds to the lossless case, (38). It is assumed that $\beta$ remains unchanged in the presence of losses, which is reasonable in the low-loss case. This assumption agrees with the usual perturbational approach in calculating the attenuation constant. As a result, the term for the lossless case still satisfies (38), from which we obtain:

$$
\begin{aligned}
\alpha & =-R_{s 1} \frac{\partial \operatorname{det}[\overline{\boldsymbol{Z}}]}{\partial X_{s 1}}\left(\frac{\partial \operatorname{det}[\overline{\boldsymbol{Z}}]}{\partial \beta}\right)^{-1} \\
& =R_{s 1} \frac{\partial \beta}{\partial X_{s 1}} .
\end{aligned}
$$

If the relation between $\beta$ and $X_{s 1}$ is already known the last expression can be used. Otherwise it is advantageous to use the expression with the differentials of det. These differentials correspond to the zero-loss case, which implies that only real operations are involved in the calculation of $\alpha$. For compactness of notation we will use the last expression of (40) hereafter. If necessary, $\partial \beta / \partial X_{s 1}$ can be calculated on the basis of the determinant.

Relation (40) is also useful in determining the kinetic inductance $L_{K}$ of structures with thin superconducting strips, which is not only dependent on $X_{s 1}$, but also on the current density distribution, cf. equation (29). Only for a uniform current density, $L_{K}$ is given by (33):

$$
L_{S}=\frac{\mu_{0} \lambda^{2}}{s t}=\frac{X_{s 1}}{\omega s},
$$

where $s$ denotes the width of the strip. Generally $L_{K}$ will be larger. To establish a relation between (40) and $L_{K}, \alpha$ is written as:

$$
\alpha=\frac{\bar{P}_{d}}{2 \bar{P}_{f}}=\frac{\frac{1}{2} R_{s 1} \int_{\text {strips }}|J|^{2} d S}{I^{2} Z_{0}} .
$$

Here $\bar{P}_{d}$ is the time-averaged dissipated power in the strips, $\bar{P}_{f}$ is the time-averaged power transmitted through the structure, $Z_{0}$ is the characteristic impedance defined on the basis of power $\bar{P}_{f}$ and current $I$ through one of the strips. In principle any of the strips can be used as the reference strip for the definition of $L_{S}$ and $Z_{0}$. It is common practice to use the strip which carries the signal for this purpose. In cases where it is not possible to identify the signal strip uniquely (e.g., slotlines) this can lead to a somewhat unconventional definition of $Z_{0}$. However the analysis remains valid as long as the same strip is used as the reference in $L_{S}$ and $Z_{0}$. Comparing (42) with the definition of $L_{K},(29)$, and using (40) yields $\left(J_{n} \ll J_{s}\right)$ :

$$
\frac{L_{K}}{L_{S}}=2 s Z_{0} \frac{\partial \beta}{\partial X_{s 1}} \text {. }
$$

In $L_{K}$ the contribution of all strips is included. The walls of the box are assumed to be perfectly conducting.
As an illustrative example of the foregoing we consider the case of the parallel-plate transmission line, which can be treated analytically [21]. For simplicity it is assumed that one of the plates is perfectly conducting. The separation between the plates is denoted by $d$, the permittivity by $\varepsilon$. Then, for $t \ll \lambda, \delta$, (18) of Swihart [21] reads as:

$$
\beta=\omega\left(\varepsilon \mu_{0}\right)^{1 / 2} \sqrt{1+X_{s 1} / \omega \mu_{0} d} .
$$

Using $R_{s 1}=\left(\omega \mu_{0}\right)^{2} \lambda^{4} \sigma_{n} / t$ ((14), (16) and (24)), evaluation of (40) yields:

$$
\alpha=\frac{\omega^{2}}{2 d}\left(\varepsilon \mu_{0}\right)^{1 / 2} \frac{\mu_{0} \sigma_{n} \lambda^{4} / t}{\sqrt{1+\lambda^{2} / d t}},
$$

which is in agreement with (37) of Swihart. Even in this analytical example it is profitable that we arrive at the correct expression for $\alpha$, without manipulating complex numbers. Also, with the help of (43), we readily obtain: $L_{K} / L_{S}=1$, as it should be for an uniform current distribution.

Concluding, the dependence of the complex propagation constant of different wave-guiding structures on the surface impedance of the strips can be evaluated by means of (38) and (40), without the numerical use of complex numbers. Equation (43) describes an efficient way of calculating the kinetic inductance of the structure.

\section{NUMERICAL RESULTS}

The modified spectral domain method, as formulated in Section II-B, is applied for a full wave analysis of the propagation characteristics of coplanar waveguide (Fig. 2). Particularly the dependence of the complex propagation constant and the kinetic inductance on the surface impedance will be analyzed. We also pay attention to the convergence of the numerical solution.

The walls of the box are perfectly conducting, whereas the thickness of the superconducting strips is much smaller than the penetration depth and the skin depth: $t \ll \lambda, \delta$. The components of the electric field in the slots are expanded in terms of the basis functions of [14]. As usual, these basis functions satisfy the edge condition for perfect conductors of zero thickness. This leads to a logarithmic divergence for quantities which involve integration of the current density squared, i.e., the attenuation constant $\alpha,(42)$, and the kinetic inductance $L_{K},(29)$. Since both quantities play an important role, and because it is easy to overlook a logarithmic divergence, a critical investigation of the convergence of the numerical solution is necessary.

The convergence is studied as a function of the number of basis function terms $M$ and as a function of the number of Fourier terms $N$. The required number $M$ is mainly dependent on the extent to which the basis functions resemble the actual field pattern. Evidently, fast variations of the basis functions, such as the divergence near the edge, are better approximated by increasing $N$.

As usual no problems are encountered in finding the wave number $\beta$, if the surface impedance is neglected. This case corresponds to solving (38) with $X_{s 1}=0$. Fig. 3(a) shows the solution of the wave number $\beta$, normalized to the wave number $k_{0}$ in free space, for different $M$ and $N$. Values of 


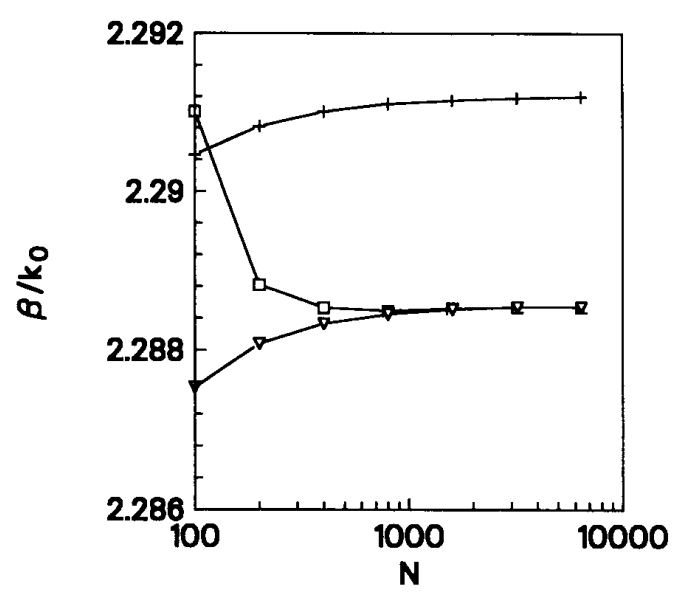

(a)

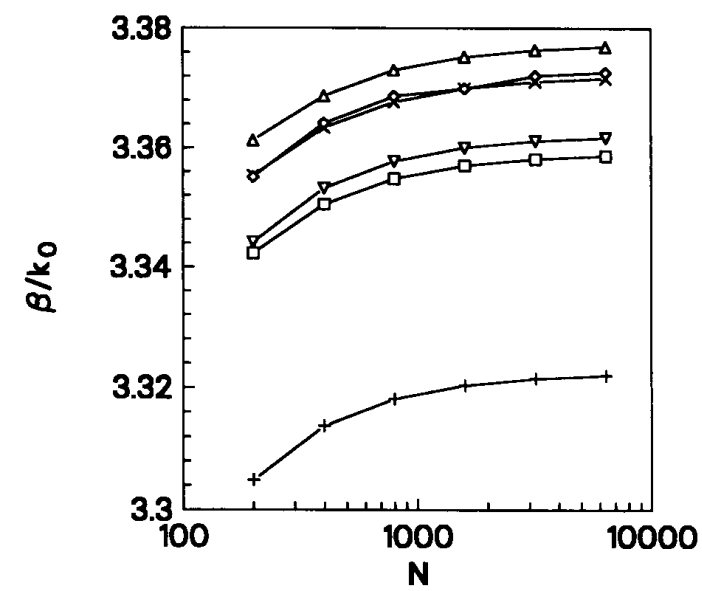

(b)

Fig. 3. The convergence of the numerical solution as a function of the number of Fourier terms $N$ for different values of the number of basis function terms $M: M=1(+), 2(\square), 3(\nabla), 4(\times), 5(\diamond)$, and $6(\Delta)$. Shown are the results of the normalized wave number $\beta / k_{0}$ for $L_{S}=0$ (a) and $L_{S}=300$ $\mathrm{nH} / \mathrm{m}$ (b).

$M$ higher than 3 give practically the same results as $M=2$ and $M=3$ for even and odd $M$ respectively. The solution converges to a limiting value, which is constant for $M>1$. Using $M=1$ and $N=100$ introduces only an error of $0.1 \%$. It turns out that the actual functional dependence of the basis functions is of little importance, as long as they incorporate the edge condition and satisfy the correct symmetry of the field pattern. These satisfactory results are in fact the justification for this established choice of the basis functions.

For $X_{s 1} \neq 0$ the divergence near the edge is smeared out as a result of the finite penetration depth. Hence, the basis functions wilich satisfy the edge condition are less suited to describe the field pattern. Therefore the convergence of $\beta / k_{0}$ with increasing $M$ is slower (Fig. 3(b)), in comparison with the case of $X_{s 1}=0$. However the solution does converge as a function of both $M$ an $N$. An increase in $M$ is more effective than the same increase in $N$. A reasonable approximation of the solution is obtained using $M=4$ and $N=400$ to 800 .

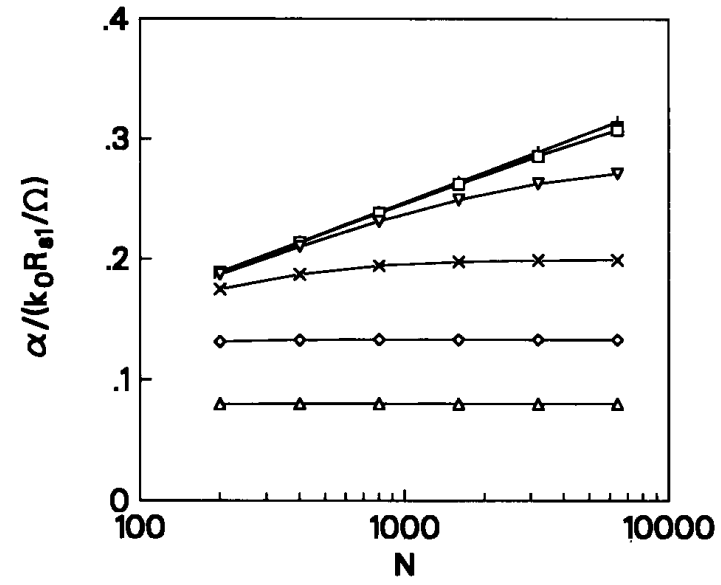

Fig. 4. The convergence of the numerical results for $\alpha$ with increasing number of Fourier terms $N$ for the different values of $L_{S}: L_{S}=0(+) .0 .03(\square) .0 .3(\nabla), 3(\times) .30(\diamond)$, and $300 \mathrm{nH} / \mathrm{m}(\Delta)$. The calculations are performed with four basis function terms $(M=4)$.

The attenuation constant is calculated according to equation (40) for different values of $L_{S}=X_{s 1} / \omega s$ (Fig. 4). For $L_{S}=0$ the results clearly exhibit a logarithmic divergence, as has to be expected. Of course the logarithmic divergence is a mathematical artefact. In reality the current density will be peaked near the edges, but never divergent. In general a practical argument is used to overcome this problem. It is stated that the relatively slow divergence is not a serious problem in view of the limited experimental accuracy in measuring $\alpha$. Indeed, reasonable values of $\alpha$ are obtained [18], but the arbitrariness in the choice of $N$ is questionable. On the other hand it can be argued that the peaked current densities make any kind of analysis very sensitive to small deviations from the model, such as material imperfections and geometrical tolerances.

The rate of convergence steadily increases for higher values of $L_{S}$ and $\lambda$ has an increasing effect in smearing out the divergence near the edges. Because the current density becomes less peaked with increasing $L_{S}$ the value of $\alpha$ decreases as well. For very small values of $L_{S}$ the value of $\alpha$ is bounded as $N$ goes to infinity. However, it seems unrealistic that for instance as many as $10^{5}$ Fourier terms are needed to obtain the correct $\alpha$. Presumably the validity of such a calculation breaks down because of a small amount of losses. Thus theoretically the calculation of $\alpha$ presents no problem for small $L_{S}$, since $\alpha$ is bounded. However practically problems are encountered in much the same way as in the case of $L_{S}=0$. It is clear from Fig. 4 that this ambiguity in the calculation of $\alpha$ ceases to exist for higher values of $L_{S}$. As we will see, this corresponds exactly to the case of experimental interest.

By virtue of (40), the slow convergence of $\alpha / R_{s 1}$ for small $L_{S}$ also causes $\beta$ to be slowly convergent with $N$. This is confirmed if we take a close look at the results for $\beta$. However, small values of $L_{S}$ cause a small change in $\beta$. Hence the slow convergence of this small change is only observed at a scale which is far beyond any practical demand of accuracy. For higher values of $L_{S}\left(=X_{s 1} / \omega s\right)$ the quantity $\partial \beta / \partial X_{s 1}$ can 


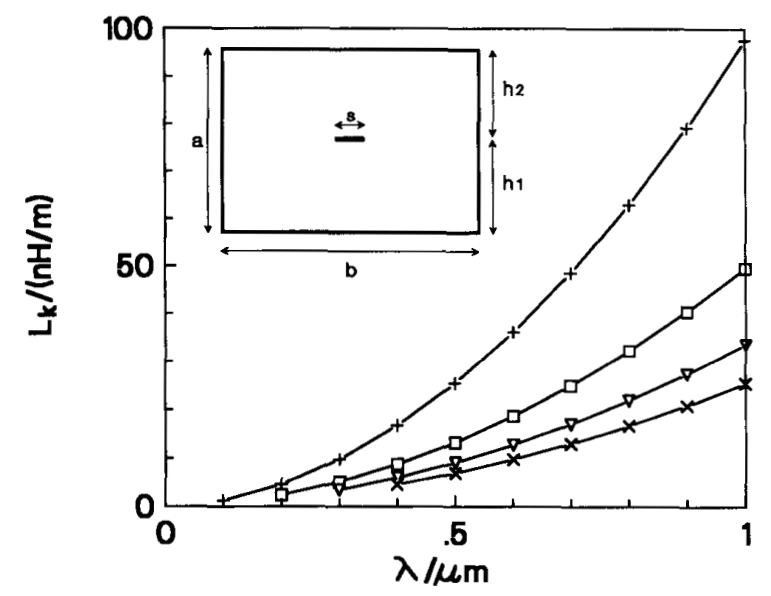

Fig. 5. The kinetic inductance $L_{K}$ of a stripline (see inset) as a function of the penetration depth $\lambda$ for different strip thicknesses $t(M=4, N=800): t=0.1(+), 0.2(\square), 0.3(\nabla), 0.4 \mu \mathrm{m}(\times)$. The walls of the box are assumed to be perfectly conducting. The geometrical parameters are: $a=846 \mu \mathrm{m}, b=2000 \mu \mathrm{m}, s=150 \mu \mathrm{m}$, and $h_{1}=h_{2}=432 \mu \mathrm{m}$

be integrated to obtain $\beta$, which means that the resultant rate of convergence of $\beta$ for a specific value of $L_{S}$ is an accumulation of the rate of convergence of $\alpha$ for smaller values of $L_{S}$. This explains why $\beta$ is converging slower with the number of Fourier terms $N$ as $L_{S}$ increases (Fig. 3).

In order to test the validity of the formulation of Section IIB for calculating $\alpha$ and $L_{K}$, we will compare our results with two other works. Sheen et al. [22] have analyzed a stripline configuration with a metallization of arbitrary thickness. The conductors, which obey the two-fluid model, are subdivided in small patches. From the transmission line equation of the resultant system of coupled transmission lines, the current distribution, resistance and inductance of the transmission line are calculated. We can use the Green's function of a coplanar waveguide for an analysis of the stripline configuration by setting $w=1 / 2(b-s)$ and $\varepsilon_{r}=1$. As mentioned before, the walls are assumed to be perfectly conducting. This is in contrast to the model of [22], in which the top and bottom wall are superconducting and the sidewalls are not present. However, the main contribution to the kinetic inductance $L_{K}$ will arise from the central strip, since the currents are most strongly peaked here. The influence of the sidewalls can be neglected, provided the distance $b$ between these wails is not too small. To limit the number of Fourier terms needed we have performed the calculations with $b=2000 \mu \mathrm{m}$. The results for $L_{K}$ on the basis of (43) are presented in Fig. 5 as a function of the penetration depth $\lambda$ for different thicknesses $t(\lambda>t)$ of the central strip. Despite the differences in geometry the agreement with the results of Sheen et al. [22] (Fig. 6) is good. This strongly indicates the validity of both methods, as both are based on a different computational scheme.

Pond et al. [5] have calculated the complex propagation constant $\gamma$ of microstrip lines with very thin superconductors $(t \ll \lambda)$, by means of solving equation (37). No numerical operations in the complex plane are necessary if we use the alternative expression (40). The results for $\alpha$. based on

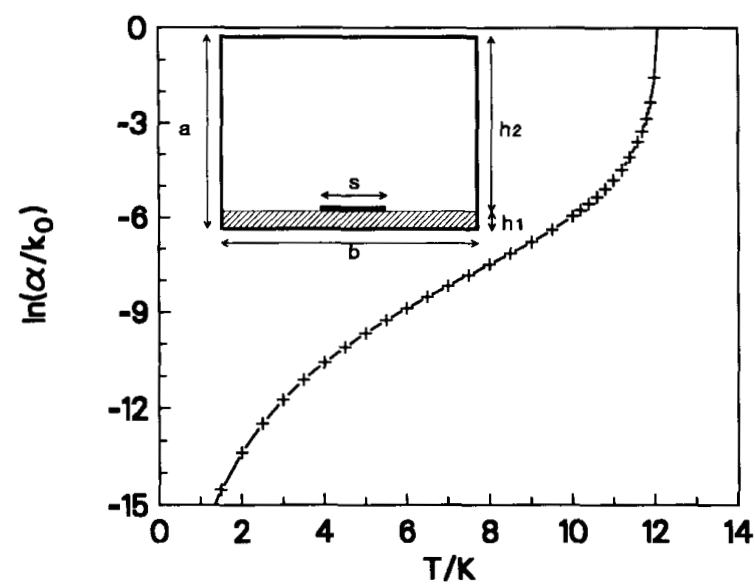

Fig. 6. The normalized attenuation constant of a microstrip line (see inset) with a strip of thickness $t=14.5 \mathrm{~nm}$ at a frequency $f=1$ $\mathrm{GHz}(M=4 . N=800)$. The microstrip line is enclosed in a perfectly conducting box. The geometrical parameters are: $\varepsilon_{r}=10.5$, and $a=b=100 \mu \mathrm{m}, s=25 \mu \mathrm{m} . h_{1}=2.25 \mu \mathrm{m}$, and $h_{2}=97.75 \mu \mathrm{m}$. The two-fluid model of Gorter and Casimir is used with $T_{c}=12.15 \mathrm{~K}$, $\lambda_{0}=320 \mathrm{~nm}$, and $\sigma_{n c}=10^{6}(\Omega \mathrm{m})^{-1}$.

(40), are shown in Fig. 6 and are seen to be in excellent agreement with Fig. 9 of [5]. Again a different configuration is handled within the framework of coplanar waveguide, this time by setting $w=1 / 2(b-s)$ and $h_{1} \rightarrow 0$. In order to make the results directly comparable, we have calculated the absolute value of $\alpha$ by inserting the dependences of the twofluid model of Gorter and Casimir, $\sigma_{n}=\sigma_{n c}\left(T / T_{c}\right)^{4}$ and $\lambda=\lambda_{0} /\left(1-\left(T / T_{c}\right)^{4}\right)^{1 / 2}$, in $R_{s 1}=\left(\omega \mu_{0}\right)^{2} \lambda^{4} \sigma_{n} / t$. The conductivity just above the critical temperature is denoted by $\sigma_{n c}$, the zero-temperature penetration depth by $\lambda_{0}$.

The foregoing demonstrates that the results of the formulation described in Section II-B and those of other methods are similar. For everyday use it is convenient to have the disposal of an empirical relation between the surface impedance and the propagation constant. For this purpose a description of $L_{K} / L_{S}$ as a function of $L_{S}$ is sufficient:

$$
\frac{L_{K}}{L_{S}}=F\left(L_{S}\right)
$$

Fig. 7 shows the numerical results of $L_{K} / L_{S}$, calculated according to (43), for different values of $w$. These data are very well represented by the function:

$$
F\left(L_{S}\right)=A+B e^{-\left\{0.14 \cdot \ln \left(50 L_{S}[\mathrm{nH} / \mathrm{m}]\right)\right\}^{C}}
$$

where $A, B$, and $C$ are geometry-specific fitting parameters. For quasi-TEM modes the parameters $A, B$, and $C$ depend only weakly on the dielectric constant (and therefore $h_{2}$ ) and frequency, because the dielectric constant has little effect on the inductance and because the dispersion is relatively low for these modes. The parameters also depend on the number of Fourier terms $N$. However, in the cases of practical interest, meaning an appreciable magnitude of $L_{S}$, the value of $F\left(L_{S}\right)$ is changing slowly with $N$. 
A transmission line model is applicable for structures, which support a quasi-TEM mode (e.g., microstrip lines and coplanar waveguides). From such a model we obtain:

$$
\beta=\beta_{0}\left(\frac{L_{K}}{L}+1\right)^{1 / 2},
$$

with the wave number $\beta_{0}$ of the structure with perfect metallization $\left(L_{S}=0\right)$, and the geometrical inductance $L=$ $\left(\beta_{0} / k_{0}\right) Z_{0} / c_{0}$, which is only moderately dependent on $L_{S} ; c_{0}$ is the phase velocity in free space. Closed-form expressions are available for the wave number $\beta_{0}$ and the characteristic impedance $Z_{0}$ [23], [24]. From (48) it also is clear that the influence of $L_{K}$ is only significant if it is an appreciable portion of $L$.

The equations (46) to (48) establish a relation between $\beta$ and $L_{S}$ or, equivalently, $X_{s 1}$. Then, invoking (40), $\alpha / R_{s 1}$ is also known for a particular $X_{s 1}$.

\section{EXPERIMENTAL RESULTS}

We have measured the resonance frequency $f_{0}$ and the unloaded quality factor $Q_{0}$ of $\mathrm{YBa}_{2} \mathrm{Cu}_{3} \mathrm{O}_{7-x}$ coplanar waveguide resonators, as described in [25]. The measurements are performed in the temperature range of 4.2 to $80 \mathrm{~K}$ at excitation levels for which the data are independent of incident microwave power $(-30 \mathrm{dBm})$. The thin films are deposited on sapphire substrates by off-axis rf magnetron sputtering [26]. A $c$-axis oriented $\mathrm{YBa}_{2} \mathrm{Cu}_{3} \mathrm{O}_{7-x}$ layer is separated from the substrate by a $\mathrm{PrBa}_{2} \mathrm{Cu}_{3} \mathrm{O}_{7-x}$ buffer layer, both layers having a thickness of $50 \mathrm{~nm}$. The buffer layer blocks the interdiffusion between $\mathrm{YBa}_{2} \mathrm{Cu}_{3} \mathrm{O}_{7-x}$ and sapphire effectively [27]. Moreover, the $\mathrm{PrBa}_{2} \mathrm{Cu}_{3} \mathrm{O}_{7-x}$ material has similar lattice constants and the same perovskite structure as $\mathrm{YBa}_{2} \mathrm{Cu}_{3} \mathrm{O}_{7-x}$, resulting in an improved lattice-match.

The normalized wave number $\beta / k_{0}=c_{0} / 2 l f_{0}$ follows from the resonance frequency $f_{0}$, the length $l$ of the resonator, and the phase velocity $c_{0}$ in free space. The relevant length of the resonator is slightly different from its physical length (5.4 $\mathrm{mm}$ ) by open-end effects. The wave number $\beta$ is influenced by the field penetration into the superconductor or by the kinetic inductance of the transmission line. From the numerical analysis of Section III we have an empirical relation between $\beta$ and $L_{S}=\mu_{0} \lambda^{2} / s t$, which can be inverted to obtain $\lambda$ from $\beta$. We are dealing with small values of $L_{S} / L$ of the order of a few percent; the geometrical inductance is evaluated as $L=\left(\beta_{0} / k_{0}\right) Z_{0} / c_{0}=377 \mathrm{nH} / \mathrm{m}(s=0.5 \mathrm{~mm}, w=0.2 \mathrm{~mm})$, using the closed-form expressions of [24], and $L_{S}$ is estimated as $L_{S}=\mu_{0} \lambda^{2} / s t \approx 2 \mathrm{nH} / \mathrm{m}$ for $\lambda \approx 200 \mathrm{~nm}, s=0.5 \mathrm{~mm}$, and $t=50 \mathrm{~nm}$. If $L_{S}$ is only a small fraction of $L$, relatively small changes in $\beta$ correspond to significant changes in $L_{S}$, cf. (48). This illustrates the difficulty that for small $L_{S} / L$ both the wave number for $L_{S}=0, \beta_{0}$, and the length $l$ of the resonator need to be known with high accuracy. The former quantity $\left(\beta_{0}\right)$ is not known experimentally $\left(L_{S} \neq 0\right.$ for $\left.T \rightarrow 0\right)$, while it is non-trivial to calculate the latter $(l)$ accurately, since this involves open-end effects. Moreover, there are more sources of uncertainty, such as the dielectric anisotropy of sapphire [28].

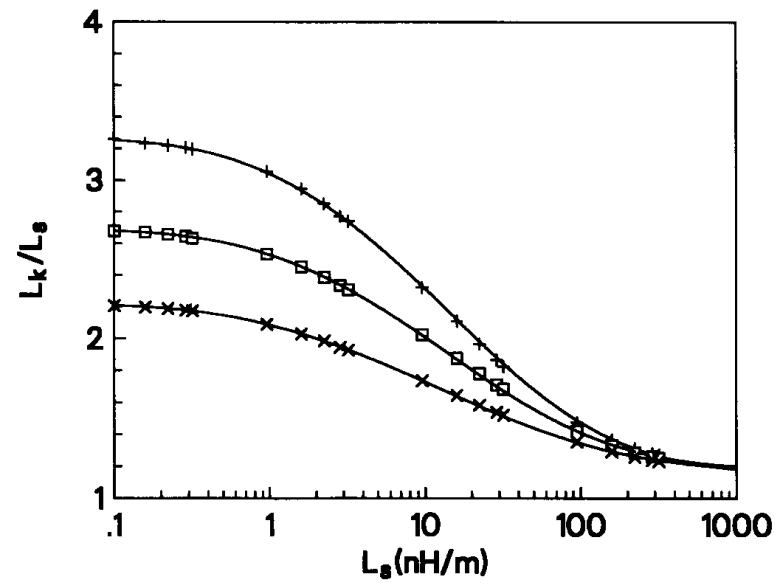

Fig. 7. The normalized kinetic inductance $L_{K} / L_{S}$ as a function of $L_{S}$ for coplanar waveguides (Fig. 2). The data points are the spectral domain results for $w=0.1(+), w=0.2(\square)$, and $w=0.5 \mathrm{~mm}(x)$. The lines are the result of a fit to (47) with parameters: $A=1.164, B=2.096$, and $C=3.666(w=0.1 \mathrm{~mm}) ; A=1.188, B=1.497$, and $C=3.661(w=0.2 \mathrm{~mm}) ; A=1.173, B=1.042$, and $C=3.362(u=0.5 \mathrm{~mm})$.

Therefore a more practical procedure is applied to determine $\beta_{0}$ and 1. The results for $\lambda$ are fitted to the temperature dependence of the two-fluid model of Gorter and Casimir, $\lambda=$ $\lambda_{0} /\left(1-\left(T / T_{c}\right)^{4}\right)^{1 / 2}$, with the free parameters $l, \lambda_{0}$, and $T_{c}$. Such a self-consistent treatment of the data is commonly used, even in cases of a small geometry (delay lines), where much higher values of $L_{S} / L$ are possible [29]. The procedure turns out to be satisfactory, since slightly different values of $\beta_{0}$ lead to somewhat different values of $l$, but to practically the same $\lambda_{0}$. The values of $A, B$, and $C$ from the empirical relation (47) are not very critical as well. We use $\beta_{0} / k_{0}=2.3367$, which is the spectral domain result for $s=0.5 \mathrm{~mm}, w=0.2 \mathrm{~mm}$, $h_{2}=1.135 \mathrm{~mm}, \varepsilon_{r}=10$, and $f=10 \mathrm{GHz}$. The value of $h_{2}$ follows from the thickness of the sapphire substrate $(0.5 \mathrm{~mm})$, which is surface mounted on an alumina motherboard ( $25 \mathrm{mil})$. The parameters $A, B$, and $C$ are only weakly dependent on $h_{2}$, as mentioned before, which implies that we can use the values shown in Fig. $7\left(h_{2}=0.635 \mathrm{~mm}\right): A=1.188, B=1.497$, and $C=3.661$. Within the same approximation we can ignore the difference between the frequency used in the calculations $(10 \mathrm{GHz})$ and the observed resonance frequency $(\approx 11 \mathrm{GHz})$. In this way we obtain $l=5.695 \mathrm{~mm}, \lambda_{0}=250 \mathrm{~nm}$, and $T_{c}=86 \mathrm{~K}$. The critical temperature $T_{c}$ is consistent with dc-resistance measurements.

The temperature dependence of the inferred $\lambda$ is examined in more detail by plotting the relative change $(\lambda(T)-\lambda(0)) / \lambda(0)$ versus temperature (Fig. 8). Also shown are the temperature dependencies of the two-fluid model (solid) and the MattisBardeen (MB) theory [9], for two different values of the energy gap parameter: $2 \Delta_{0} / k T_{c}=3.5$ (dashed) and $2 \Delta_{0} / k T_{c}=4.8$ (dot-dashed). The MB temperature dependence of $\lambda$ changes slowly with the electron mean free path $l$. The curves shown in Fig. 8 correspond to $l / \pi \xi_{0}=2$. The MB theory is used in the local limit [30], where the field variation across the size of the Cooper pairs can be neglected, because the zero-temperature 


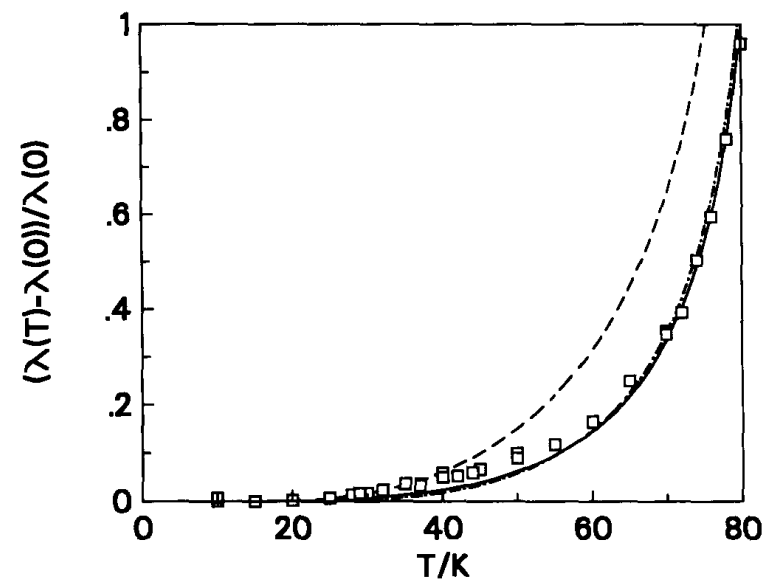

Fig. 8. Experimental results of the relative change of the penetration depth $(\lambda(T)-\lambda(0)) / \lambda(0)$, with temperature $T(\square)$. The curves represent the two-fluid model (solid), and the MB theory with $l / \pi \xi_{0}=2 . \lambda_{0}=140$ $\mathrm{nm}$, and the gap parameters $2 \Delta_{0} / k T_{c}=3.5$ (dashed) and $2 \Delta_{0} / k T_{c}=4.8$ (dot-dashed).

coherence length $\xi_{0}$ is much smaller than the penetration depth $\lambda_{0}$ at zero temperature. This limit is appropriate for hightemperature superconductors, since $\xi_{0}$ is of the order of a few $\mathrm{nm}$ in these materials, while $\lambda_{0}$ is of the order of several hundred $\mathrm{nm}$. Less clear is the validity of isotropic pairing, as is assumed in the MB theory. However, a detailed picture of the coupling in high-temperature superconductors is not available.

The experimental results agree with a gap parameter $2 \Delta_{0} / k T_{\mathrm{c}}=3.5$ for lower temperatures $\left(T \lesssim 0.5 T_{c}\right)$, while the data for $T \gtrsim 0.5 T_{\mathrm{c}}$ are better represented by $2 \Delta_{0} / k T_{\mathrm{c}}=$ 4.8. The value of 3.5 agrees with the BCS weak-coupling limit [31], the value of 4.8 is comparable to results of other works, where values of 4.3 to 5 are reported for the same temperature range [6], [32], [33]. The two-fluid model closely resembles the $\mathrm{MB}$ theory for $2 \Delta_{0} / k T_{c}=4.8$, despite the usual application of this model to superconductors in the weakcoupling limit $\left(2 \Delta_{0} / k T_{c}=3.53\right)$. This is caused by the fact that the zero-temperature penetration depth $\lambda_{0}$ in the two-fluid model should be somewhat higher than $\lambda_{0}$ in the BCS weakcoupling limit in order to obtain agreement for temperatures near $T_{c}$.

Thus the results over the entire temperature range are not consistent with a single-gap BCS temperature dependence, which confirms the results of [6]. Possibly this can be attributed to the presence of weak links in the material [34], [35], which also explains the reduced critical temperature $T_{c}=86$ $\mathrm{K}$ and the increased penetration depth $\lambda_{0}=250 \mathrm{~nm}$, compared to the more or less established intrinsic value $\lambda_{I 0}=140 \mathrm{~nm}$ [6], [33]. The influence of weak links is accounted for in a model of Hylton et al. [36], which predicts that the resultant penetration depth $\lambda=\left(\lambda_{I}^{2}+\lambda_{J}^{2}\right)^{1 / 2}$ is modified by an effective penetration depth $\lambda_{J} \propto I_{c}^{-1 / 2}$ of the weak lines alone. If we take the weak-link critical current temperature dependence of Ambegaokar-Baratoff [37], $I_{\mathcal{c}} \propto\left(\Delta / \Delta_{0}\right) \tanh (\Delta / 2 k T)$, the temperature dependence of $\lambda_{J}$ is the same as the dominant low-temperature MB temperature dependence of $\lambda_{I}$. Therefore

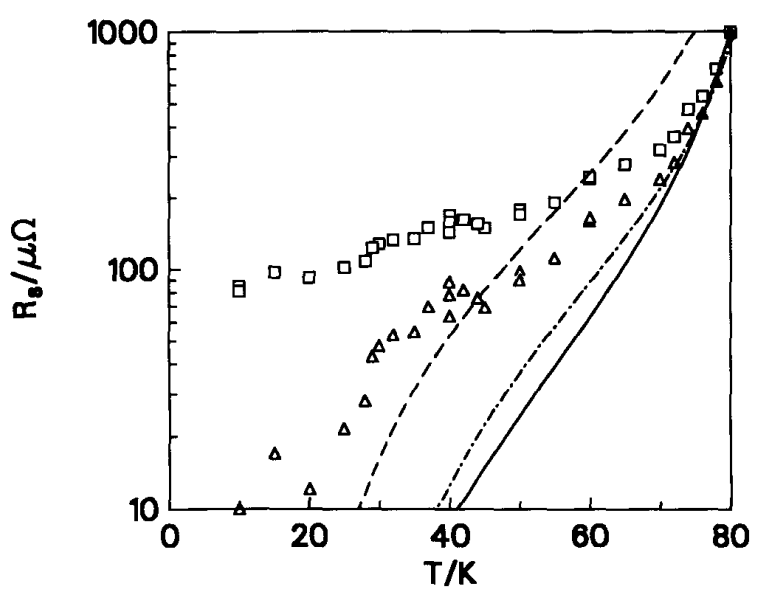

Fig. 9. Experimental results of the surface resistance $R_{s}$, absolute ( $\square$ ) and relative to zero-temperature $(\Delta)$. The curves represent the two-fluid model (solid), and the $\mathrm{MB}$ theory with $l / \pi \xi_{0}=2 \cdot \lambda_{0}=140 \mathrm{~nm}$, and the gap parameters $2 \Delta_{0} / k T_{c}=3.5$ (dashed) and $2 \Delta_{0} / k T_{c}=4.8$ (dot-dashed).

this temperature dependence does not improve the fit of the experimental results to a single-gap dependence. However, the precise change of $I_{c}$ with temperature is open to discussion.

Next we turn to the results of $R_{s}$, which are deduced from the data $Q_{0}$. By virtue of $Q_{0}=\beta / 2 \alpha$ and (40) we have; $R_{s}=\beta t / 4 \lambda Q_{0} \partial \beta / \partial X_{s 1}$, where $R_{s}$ is the surface resistance of bulk material, cf. (24). With the application of the empirical relations (46) to (48) between $\beta$ and $L_{S}$ (or $\lambda$ ), $R_{s}$ can be calculated from $Q_{0}$, if $\lambda$ is known. In this case we will use the two-fluid model of Gorter and Casimir to determine $\lambda$, which is reasonable over the entire temperature range (Fig. 8).

Fig. 9 shows the results for $R_{s}$ as a function of temperature ( $\square$ ). As usual, $R_{s}$ decreases rapidly as the temperature is reduced below $T_{c}$. For lower temperatures $R_{s}$ approaches a constant value, which is an indication of non-intrinsic behavior. The most likely reasons of this residual $R_{s}$ are the losses in the weak links [34], [35] or the dielectric losses [38]. In any event, the low-temperature value $R_{s}=80 \mu \Omega$ is much lower than the textbook value of OFHC copper in the same frequency and temperature range, $R_{s}=5 \mathrm{~m} \Omega$. This illustrates the suitability of these films for microwave applications with a high demand on $R_{s}$, such as delay lines and high-performance filters. Both the residual $R_{s}$ and $T_{c}$ are in remarkable agreement with the results of thin films on sapphire with a $\mathrm{SrTiO}_{3}$ buffer layer, reported by Char et al. [39] $\left(R_{s}=65 \mu \Omega\right.$ at $10 \mathrm{GHz}$, which would imply $R_{s}=80 \mu \Omega$ at $11 \mathrm{GHz}$ according to a quadratic frequency dependence, and a superconducting transition at $87 \mathrm{~K}$ with a $1 \mathrm{~K}$ transition width). Also shown in Fig. 9 are the results for $R_{s}$ with the zero-temperature value subtracted, $R_{s}(T)-R_{s}(0),(\Delta)$. The aim of this operation is to cancel the non-intrinsic losses, although the temperature dependence of these losses is not necessarily negligible. The data of $R_{s}(T)-R_{s}(0)$ at the lowest temperatures should be interpreted with some caution, since the relative errors become large for small differences.

The solid curve represents the two-fluid model with the parameters $\lambda_{0}=250 \mathrm{~nm}$ and $\sigma_{n c}=3 \cdot 10^{6}(\Omega \mathrm{m})^{-1}$, where 
$\sigma_{n c}$ is the conductivity just above $T_{c}$. The other two curves are the results of the MB theory for $l / \pi \xi_{0}=2, \lambda_{0}=140 \mathrm{~nm}$, and gap values $2 \Delta_{0} / k T_{c}=3.5$ (dashed) and $2 \Delta_{0} / k T_{c}=4.8$ (dotdashed). The calculations are performed at $11 \mathrm{GHz}$; the $2 \%$ change of the observed resonance frequency with temperature is neglected. In order to match the two-fluid model and the MB theory near $T_{c}$, we need a higher value of $\sigma_{n c}$ at $11 \mathrm{GHz}$ than Drabeck et al. [32] at $100 \mathrm{GHz}\left(1.5 \cdot 10^{-6}(\Omega \mathrm{m})^{-1}\right)$. This is caused by the additional MB frequency dependence $\ln (k T / \hbar \omega)$ of the surface resistance $(\hbar \omega \ll k T)$. which illustrates that we should not take the value of $\sigma_{n c}$ too serious. The experimental results close to $T_{c}$ are well represented by $2 \Delta_{0} / k T_{c}=4.8$. In this temperature range the value of $l / \pi \xi_{0}=2$ is found to give the best agreement with the experimental results. The data at lower temperatures are better described by the other gap parameter $2 \Delta_{0} / k T_{c}=3.5$. although it can not be concluded decisively that this is the correct value of the gap for lower temperatures. However the discrepancy with the experimental results is limited in view of the margins of error and the neglect of the influence of temperature with respect to the weak links.

\section{CONCLUSIONS}

We have analyzed the surface impedance of an infinite normal or superconducting film of arbitrary thickness. The surface reactance $X_{s}$ of very thin superconducting films $(t \lesssim$ $\lambda$ ) is increased by a factor $\lambda / t$ compared to $X_{s}$ of relatively thick films $(t \gg \lambda)$. The surface resistance $R_{s}$ and the kinetic inductance $L_{K}$ are increased by a factor $2 \lambda / t$. The analysis also shows that in the case of very thin films the boundary conditions for the field agree with the complex boundary conditions [5], which are used in a modification of the spectral domain analysis of different wave-guiding structures.

The influence of the surface impedance $Z_{s}$ on the propagation constant $\gamma=\alpha+j \beta$ is investigated by means of the modified spectral domain method. We found expressions which allow an efficient calculation of the attenuation constant $\alpha$ and the kinetic inductance $L_{K}$. An empirical model of the relation between the wave number $\beta$ and $L_{k}$ is presented, which describes the exact results very well.

This model is used to determine the penetration depth $\lambda$ and the surface resistance $R_{s}$ from the experimental results for the resonance frequency and the unloaded quality factor of $\mathrm{YBa}_{2} \mathrm{Cu}_{3} \mathrm{O}_{7-x}$ coplanar waveguide resonators. The $\mathrm{YBa}_{2} \mathrm{Cu}_{3} \mathrm{O}_{7-x}$ layers are deposited on sapphire with a $\mathrm{PrBa}_{2} \mathrm{Cu}_{3} \mathrm{O}_{7-x}$ buffer layer. The observation of a low residual surface resistance, $R_{s}=80 \mu \Omega$, supports the conclusion from other work [27], that the buffer layer prevents interdiffusion, while constituting a suitable basis for epitaxial growth of $\mathrm{YBa}_{2} \mathrm{Cu}_{3} \mathrm{O}_{7-x}$.

The results indicate that the temperature dependence of $\lambda$ and $R_{s}$ is not characterized by a single-gap BCS temperature dependence. For lower temperatures a conventional BCS weak-coupling gap value $2 \Delta_{0} / k T_{c}=3.52$ seems appropriate, while $2 \Delta_{0} / k T_{c}=4.8$ describes our findings for higher temperatures. Our results suggest an electron free mean path value $l / \pi \xi_{0}=2$. The origins of non-BCS behavior have been interpreted in terms of weak links. However, other interpretations [40] can not be ruled out, since the couplingmechanism for electrons in high-temperature superconductors is not clearly understood.

\section{ACKNOWLEDGMENT}

The authors wish to acknowledge the experimental assistance of A. E. Reitsma and are indebted to J. Gao for the thin film preparation.

\section{REFERENCES}

[1] J. L. Stone and W. H. Hartwig, "Performance of superconducting oscillators and filters," J. Appl. Phys., vol. 39, pp. 2665-2671, 1968.

[2] S. H. Talisa, M. A. Janocko, C. Moskowitz, J. Talvacchio, J. F. Billing, R. Brown, D. C. Buck, C. K. Jones, B. R. McAvoy, G. R. Wagner, and D. H. Watt. "Low- and high-temperature superconducting microwave filters," IEEE Trans. Microwave Theory Tech., vol. 39, pp. 1448-1454, 1991.

[3] J. M. Pond, J. H. Claassen, and W. L. Carter, "Measurements and modeling of kinetic inductance microstrip delay lines," IEEE Trans. Microwave Theory Tech., vol. MTT-35, pp. 1256-1262, 1987.

[4] R. S. Withers, A. C. Anderson, P. V. Wright, and S. A. Reible, "Superconductive tapped delay lines for microwave analog signal processing," IEEE Trans. Magn.. vol. MAG-19, pp. 480-484, 1983.

[5] J. M. Pond, C. M. Krowne, and W. L. Carter, "On the application of complex resistive boundary conditions to model transmission lines consisting of very thin superconductors," IEEE Trans. Microwave Theory Tech., vol. 37, pp. 181-190, 1989.

[6] S. M. Anlage, B. W. Langley, G. Deutscher, J. Halbritter, and M. R. Beasley, "Measurements of the temperature dependence of the magnetic penetration depth in $\mathrm{YBa}_{2} \mathrm{Cu}_{3} \mathrm{O}_{7-x}$ superconducting thin films," Phys. Rev. B, vol. 44, pp. 9764-9767, 1991.

[7] Z. Schlesinger, R. T. Collins, F. Holtzberg, C. Feild, G. Koren, and A. Gupta, "Infrared studies of the superconducting energy gap and normalstate dynamics of the high- $\mathrm{T}_{c}$ superconductor $\mathrm{YBa}_{2} \mathrm{Cu}_{3} \mathrm{O}_{7}$, , Phys. Rev. B. vol. 41, pp. 11237-11259, 1990.

[8] F. London and H. London, "The electromagnetic equations of superconductors," Proc. Roy. Soc. (London), vol. A149, pp. 71-88, 1935.

[9] D. C. Mattis and J. Bardeen, "Theory of the anomalous skin effect in normal and superconducting metals," Phys. Rev, vol. 111, pp. 412-417, 1958 .

[10] T. Van Duzer and C. W. Turner. Principles of Superconductive Devices and Circuits. New York: Elsevier, 1981.

$111]$ R. F. Harrington, Time-Harmonic Electromagnetic Fields. New York: McGraw-Hill, 1961.

[12] R. E. Matick, Transmission Lines for Digital and Communication Networks. New York: McGraw-Hill, 1969.

[13] R. Meservey and P. M. Tedrow. "Measurements of the kinetic inductance of superconducting linear structures," J. Appl. Phys., vol. 40, pp. $2028-2034,1969$.

[14] R. W. Jackson, "Considerations in the use of coplanar waveguide for millimeter-wave integrated circuits," IEEE Trans. Microwave Theory Tech., vol. MTT-34, pp. 1450-1456, 1986.

[15] D. Kinowski, F. Huret, P. Pribetich. and P. Kennis, "Influence des supraconducteurs sur les caractéristiques de propagation de lignes planaires pour circuits intégrés microondes," Ann. Télécommun., vol. 45, pp. $334-343,1990$.

[16] D. Nghiem, J. T. Williams, and D. R. Jackson, "A general analysis of propagation along multiple-layer superconducting stripline and microstrip transmission lines," IEEE Trans. Microwave Theory Tech., vol. 39. pp. 1553-1565, 1991.

[17] R. Mittra and S. W. Lee, Analyrical Techniques in the Theory of Guided Waves. New York: Macmillan, 1971.

[18] D. Mirshekar-Syahkal and J. B. Davies, "An accurate, unified solution to various fin-line structures, of phase constant, characteristic impedance, and attenuation," IEEE Trans. Microwave Theory Tech., vol. MTT-30, pp. 1854-1861, 1982.

[19] P. Heitk'amper and W. Heinrich, "On the calculation of conductor loss on planar transmission lines assuming zero strip thickness," IEEE Trans. Microwave Theory Tech., vol. 39, pp. 586-588, 1991.

[20] B. Bhat and S. K. Koul, Analysis, Design and Applications of Fin Lines. Norwood, MA: Artech House, 1987.

[21] J. C. Swihart, "Field solution for a thin-film superconducting strip transmission line," J. Appl Phys., vol. 32. pp. 461-469, 1961. 
[22] D. M. Sheen, S. M. Ali, D. E. Oates, R. S. Withers, and J. A. Kong, "Current distribution, resistance, and inductance for superconducting strip transmission lines," IEEE Trans. Appl. Supercond., vol. 1, pp. $108-115,1991$

[23] K. C. Gupta, R. Garg, and I. J. Bahl, Microstrip Lines and Slotlines. Dedham, MA: Artech House, 1979.

[24] G. Ghione and C. Naldi, "Analytical formulas for coplanar lines in hybrid and monolithic MICs," Electron. Lett., vol. 20, pp. 179-181, 1984.

[25] B. B. G. Klopman, H. W. Weijers, J. Gao, G. J. Gerritsma, and H. Rogalla, "Integration of semiconductor and ceramic superconductor devices for microwave applications," IEEE Trans. Magn., vol. 27, pp. 2821-2823, 1991.

[26] J. Gao, B. Häuser, and H. Rogalla, "High critical current density thin and ultra thin $\mathrm{YBa}_{2} \mathrm{Cu}_{3} \mathrm{O}_{\bar{i}-x}$ films made by a modified $\mathrm{RF}$-magnetron technique," J. Appl. Phys., vol. 67, pp. 2512-2515, 1990.

[27] W. A. M. Aamink, A. van Silfhout, and H. Rogalla, "Structure and composition of high- $T_{c}$ superconducting multi layer structures," in Mat Res. Soc. Symp. Proc.. Anaheim, CA, vol. 221, pp. 337-342, 1991

[28] P. H. Ladbrooke, "Some effects of field perturbation upon cavityresonance and dispersion measurements on MIC dielectrics," IEEE Trans. Microwave Theory Tech., vol. MTT-25, pp. 892-903, 1977.

[29] B. W. Langley, S. M. Analage, R. F. W. Pease, and M. R. Beasley, "Magnetic penetration depth measurements of superconducting thin films by a microstrip resonator technique," Rev. Sci. Instrum., vol. 62 , pp. 1801-1812, 1991

[30] J. J. Chang and D. J. Scalapino, "Electromagnetic response of layered superconductors," Phys. Rev. B, vol. 40, pp. 4299-4305, 1989

[31] G. Rickayzen, Theory of Superconductivity. New York: Interscience Publishers, 1965.

[32] L. Drabeck, K. Holczer, G. Grüner, J. J. Chang, D. J. Scalapino, and T. Venkatesan, "An experimental investigation of $\mathrm{YBa}_{2} \mathrm{Cu}_{3} \mathrm{O}_{7}$ films at millimeter-wave frequencies," J. Supercond., vol. 3, pp. 317-322, 1990

[33] S. Sridhar. D. H. Wu, and W. Kennedy, "Temperature dependence of electrodynamic properties of $\mathrm{YBa}_{2} \mathrm{Cu}_{3} \mathrm{O}_{y}$ crystals," Phys. Rev. Lett., vol. 63 , pp. $1873-1876,1989$.

[34] J. Halbritter, "On extrinsic effects in the surface impedance of cuprate superconductors by weak links," J. Appl. Phys., vol. 71, pp. 339-343, 1992.

[35] S. S. Laderman, R. C. Taber, R. D. Jacowitz, J. L. Moll, T. L. Hylton, A. F. Marshall, T. H. Geballe, and M. R. Beasley, "Resistive loss at 10 $\mathrm{GHz}$ in c-axis-aligned in-situ-grown $\mathrm{YBa}_{2} \mathrm{Cu}_{3} \mathrm{O}_{-}$films." Phys. Rev. B. vol. 43, pp. 2922-2933, 1991.

[36] T. L. Hylton, A. Kapitulnik, M. R. Beasley, J. P. Carini, L. Drabeck and G. Grüner, "Weakly coupled grain model of high-frequency losses in high $\mathrm{T}_{c}$ superconducting thin films," Appl. Phys. Lett., vol. 53, pp. $1343-1345,1988$.

[37] V. Ambegaokar and A. Baratoff, "Tunneling between superconductors," Phys. Rev. Lett., vol. 11, p. 104, 1963.

[38] V. B. Braginski, V. I. Panov, and S. I. Vasiliev, "The properties of superconducting resonators on sapphire," IEEE Trans. Magn., vol MAG-17, pp. 955-957, 1981.

[39] K. Char, N. Newman, S. M. Garrison, R. W. Barton, R. C. Taber, S. S. Laderman, and R. D. Jacowitz, "Microwave surface resistance of epitaxial $\mathrm{YBa}_{2} \mathrm{Cu}_{3} \mathrm{O}_{7}$ thin films on sapphire," Appl. Phys. Lett., vol 57. pp. 409-411, 1990.

[40] V. Z. Kresin and S. A. Wolf, "Major normal and superconducting parameters of high- $\mathrm{T}_{c}$ oxides," Phys. Rev. B, vol. 41, pp. 4278-4285, 1990.

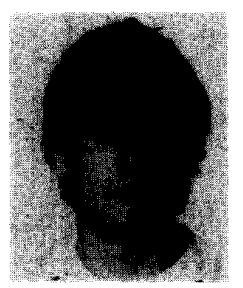

Boele B. G. Klopman was born in the Netherlands in May 1962. He received the B.S. degree in electrical engineering and applied physics in 1985, and the M.S. degree in applied physics in 1988. Pursuing the Ph.D. degree, he is currently involved in the investigation of the high-frequency properties of high-temperature superconducting thin films.

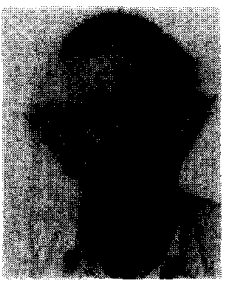

Gerrit J. Gerritsma was born in The Hague, the Netherlands, in 1943. He received his M.S. degree in applied physics in 1968 from the Technical University of Delft, the Netherlands. He became a member of the faculty of applied physics of the University of Twente, the Netherlands, in 1969. There he obtained his $\mathrm{Ph} . \mathrm{D}$. degree in applied physics in 1979.

From 1971 until 1974 he was a member of the Board of Governors of the University of Twente. He holds a position as Associate Professor in the faculty of applied physics since 1976. His current research interests are in the field of applications of high-temperature superconductivity, ranging from thin film fabrication and characterization, the fabrication of Josephson junctions and the study of their physical properties up to the design and testing of passive and active microwave and millimeter-wave circuits as well as digital electronic circuits and analog-to-digital converters.

Dr. Gerritsma is a member of the Materials Research Society (USA).

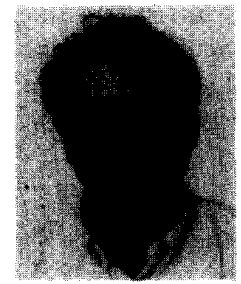

Horst Rogalla was born in Gelsenkirchen, Germany, in November 1947. He received the Ph.D. degree in 1979 in Münster, Germany. From 1977 he was member of the Faculty of Physics in Giessen, Germany. After two research stays in 1985 and 1986 at the Harvard University he habilitated in physics in 1986.

Since 1987 he has been professor for physics at the faculty of Applied Physics in Twente, the Netherlands, and chairman of the Low Temperature Division. Since 1992 he has been scientific director of the Center of Material Science of the University of Twente. His research interests are in superconducting electronics and material science, especially related to thin film growth and properties.

He is a member of the Dutch, German and American Physical Society as well as the American and European Materials Research Society. 